\title{
Branched-chain amino acids in health and disease: metabolism, alterations in blood plasma, and as supplements
}

\author{
Milan Holeček@
}

\begin{abstract}
Branched-chain amino acids (BCAAs; valine, leucine, and isoleucine) are essential amino acids with protein anabolic properties, which have been studied in a number of muscle wasting disorders for more than 50 years. However, until today, there is no consensus regarding their therapeutic effectiveness.

In the article is demonstrated that the crucial roles in BCAA metabolism play: (i) skeletal muscle as the initial site of BCAA catabolism accompanied with the release of alanine and glutamine to the blood; (ii) activity of branched-chain keto acid dehydrogenase (BCKD); and (iii) amination of branched-chain keto acids (BCKAs) to BCAAs. Enhanced consumption of BCAA for ammonia detoxification to glutamine in muscles is the cause of decreased BCAA levels in liver cirrhosis and urea cycle disorders. Increased BCKD activity is responsible for enhanced oxidation of BCAA in chronic renal failure, trauma, burn, sepsis, cancer, phenylbutyrate-treated subjects, and during exercise. Decreased BCKD activity is the main cause of increased BCAA levels and BCKAs in maple syrup urine disease, and plays a role in increased BCAA levels in diabetes type 2 and obesity. Increased BCAA concentrations during brief starvation and type 1 diabetes are explained by amination of BCKAs in visceral tissues and decreased uptake of BCAA by muscles.

The studies indicate beneficial effects of BCAAs and BCKAs in therapy of chronic renal failure. New therapeutic strategies should be developed to enhance effectiveness and avoid adverse effects of BCAA on ammonia production in subjects with liver cirrhosis and urea cycle disorders. Further studies are needed to elucidate the effects of BCAA supplementation in burn, trauma, sepsis, cancer and exercise. Whether increased BCAA levels only markers are or also contribute to insulin resistance should be known before the decision is taken regarding their suitability in obese subjects and patients with type 2 diabetes.

It is concluded that alterations in BCAA metabolism have been found common in a number of disease states and careful studies are needed to elucidate their therapeutic effectiveness in most indications.
\end{abstract}

Keywords: Cachexia, Ammonia, Glutamine, Diabetes, Cirrhosis, Nutrition

\section{Background}

The branched-chain amino acids (BCAAs), valine, leucine, and isoleucine are essential amino acids, which have been studied in a number of disorders, notably liver cirrhosis, renal failure, sepsis, trauma, burn injury, and cancer. BCAA supplementation has been thought to promote anabolic pathways and therefore mitigate cachexia, prevent or treat signs of hepatic encephalopathy, attenuate fatigue during exercise, promote wound healing, and stimulate insulin

Correspondence: holecek@lfhk.cuni.cz

Department of Physiology, Faculty of Medicine in Hradec Kralove, Charles

University, Simkova 870, 500 03, Hradec Kralove, Czech Republic production. However, until today, there is not consensus regarding their use as nutritional supplements $[1,2]$.

The intentions of this article are to: (i) review main metabolic pathways and supposed effects of BCAAs; (ii) assess the causes of alterations in metabolism and BCAA levels in various healthy and pathological conditions; and (iii) provide current views on their use as nutritional supplements for the main possible indications. As the main pathways of all three BCAAs are common and mixtures of all three BCAAs are used in most indications, the article does not describe the differences in effects of specific BCAAs.

(C) The Author(s). 2018 Open Access This article is distributed under the terms of the Creative Commons Attribution 4.0 International License (http://creativecommons.org/licenses/by/4.0/), which permits unrestricted use, distribution, and reproduction in any medium, provided you give appropriate credit to the original author(s) and the source, provide a link to the Creative Commons license, and indicate if changes were made. The Creative Commons Public Domain Dedication waiver (http://creativecommons.org/publicdomain/zero/1.0/) applies to the data made available in this article, unless otherwise stated. 


\section{BCAA metabolism}

\section{BCAA catabolism (Fig. 1)}

Unlike most amino acids, the initial step of BCAA catabolism does not take place in the liver due to low hepatic activity of branched-chain-amino-acid aminotransferase (BCAT), the first enzyme in the BCAA catabolism pathway. Therefore, the BCAA increase rapidly in systemic circulation after protein intake and are readily available to extrahepatic tissues. This phenomenon gives a unique advantage to the BCAA-based nutritional formulas compared with others, especially those targeted on muscles and brain.

The initial site of most of the BCAA catabolism is skeletal muscle because of the BCAT high activity. The BCAT reaction involves the reversible transfer of the BCAA amino group to $\alpha$-ketoglutarate $(\alpha-K G)$ to form glutamate and the corresponding branched-chain keto acids (BCKAs) , $\alpha$-ketoisocaproate (KIC, ketoleucine), $\alpha$-keto- $\beta$-methylvalerate (KMV, ketoisoleucine), and $\alpha$-ketoisovalerate (KIV, ketovaline). Glutamate then acts as an amino group source to form alanine (ALA) from pyruvate or as a substrate for ammonia detoxification to glutamine (GLN). GLN, ALA, and a significant portion of the BCKA are released from muscles to the blood.

The second enzyme of BCAA catabolism, branchedchain $\alpha$-keto acid dehydrogenase (BCKD), is a multienzyme complex located on the inner surface of the inner mitochondrial membrane, which catalyzes irreversible decarboxylation of the BCKA to the corresponding branched-chain acyl-CoA esters. The BCKD is regulated by the phosphorylation-dephosphorylation mechanism.
Phosphorylation mediated by a specific kinase results in inactivation, while dephosphorylation by a specific phosphatase activates the enzyme. Changes in kinase activity may play a main role.

The BCKD activity is highest in the liver, intermediate in kidneys and heart, and low in muscles, adipose tissue, and brain [3]. When the weights of individual tissues are taken into consideration, muscles, which make up 35 to $40 \%$ of total body weight, should contribute substantially to total body BCAA utilization. Thus, BCAA degradation is under joint control of a number of tissues, among which the muscle and liver play a dominant role (Fig. 2). Many influences including cytokines, hormones, nutrients, and various metabolites affect the activity state of the enzyme [3]. The remarkable rise in BCKD activity in muscles induces endotoxin or tumor necrosis factor alpha (TNF- $\alpha$ ) administration $[4,5]$.

Beyond the BCKD reaction, the metabolism of the BCAA diverges into separate pathways. Catabolism of $\mathrm{KIC}$ leads to acetyl-CoA and acetoacetate (KIC is ketogenic), KIV is catabolized to succinyl-CoA (KIV is glucogenic), and KMV to acetyl-CoA and succinyl-CoA (KMV is both glycogenic and ketogenic). A special product of $\mathrm{KIC}$ catabolism is $\beta$-hydroxy- $\beta$-methylbutyrate (HMB) synthesized in the reaction catalyzed by KIC dioxygenase.

\section{Amination of the BCKA and interorgan cycling of the BCAA and BCKA (Fig. 3)}

As the BCAT reaction is reversible and near equilibrium, its direction should respond to changes in concentrations

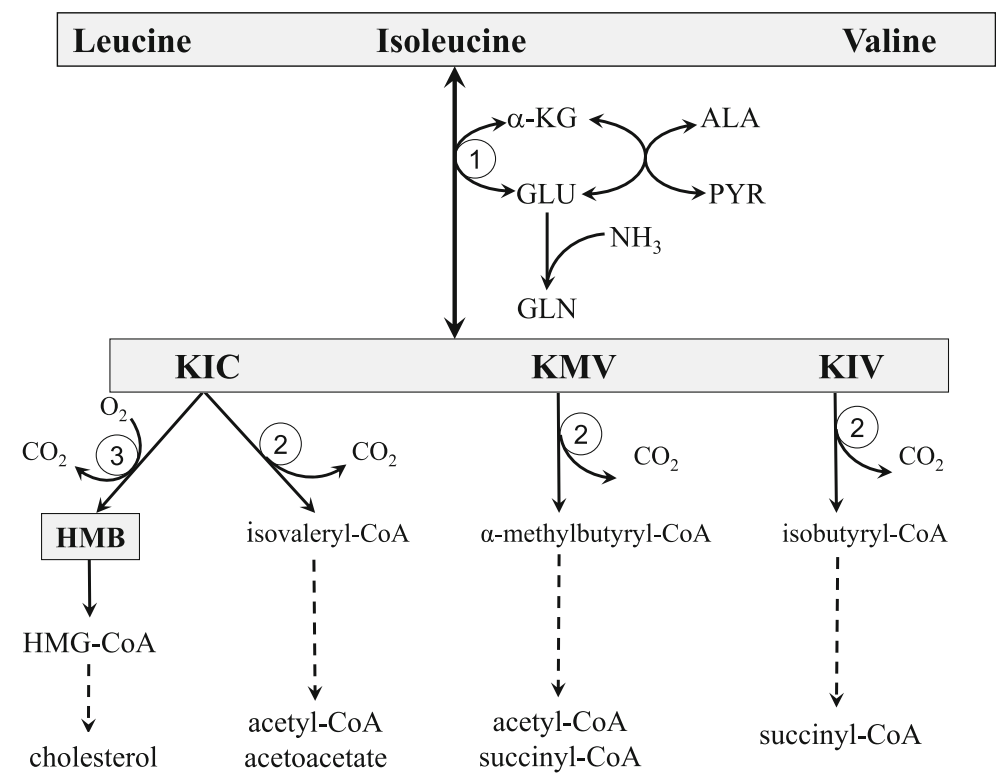

Fig. 1 Main pathways of BCAA catabolism. ALA, alanine; GLU, glutamate; GLN, glutamine; HMB, $\beta$-hydroxy- $\beta$-methylbutyrate; HMG-CoA, 3-hydroxy-3-methylglutaryl-COA; KIC, a-ketoisocaproate (ketoleucine); KIV, a-ketoisovalerate (ketovaline); KMV, a-keto- $\beta$-methylvalerate (ketoisoleucine); a-KG, a-ketoglutarate. 1, branched-chain-amino-acid aminotransferase (BCAT); 2, branched-chain a-keto acid dehydrogenase (BCKD); 3, KIC dioxygenase 


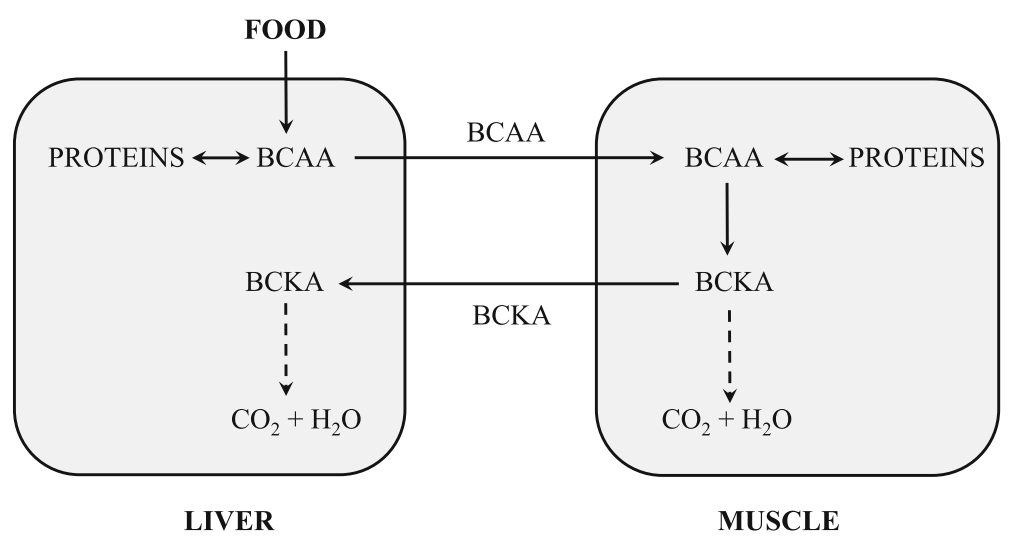

Fig. 2 Cooperation of the muscles and the liver in BCAA catabolism. BCAA, branched-chain amino acids; BCKA, branched-chain keto acids

of BCAA and BCKA, and availability of the donors and acceptors of nitrogen. In most conditions the majority of the BCAA uptake and BCKA release occurs in muscles, while amination of the BCKA to the BCAA may occur in other tissues, notably in the liver, kidneys and enterocytes. Direct evidence of the BCKA amination to the BCAAs was provided by studies using labelled BCKA showing the labelled BCAA in proteins [6]. The main sources of nitrogen for amination of the BCKA are GLN, glutamate, and ALA [3].

The studies demonstrate that BCAA synthesis from the BCKA is activated in various muscle wasting disorders, e.g. sepsis, trauma, or surgery, in which the muscles release high amounts of GLN and ALA to the blood [7-11]. A marked increase in leucine release was observed by the isolated liver of endotoxin-treated animals after addition of KIC into perfusion medium [7]; higher synthesis of the BCAA from BCKA was shown in the liver perfused with medium containing $0.5 \mathrm{mM}$ GLN when compared to perfusion with medium without GLN [8]. Amination of the BCKA may have a role in the unique increase of all three BCAA in blood plasma during a brief starvation characterized by accelerated release of ALA, GLN, and BCKA from muscles and augmented gluconeogenesis in the liver $[9,10]$.

The above-mentioned findings indicate the existence of an interorgan cycle (Fig. 3) that attenuates the loss of essential BCAA in various physiological and pathological conditions [11].

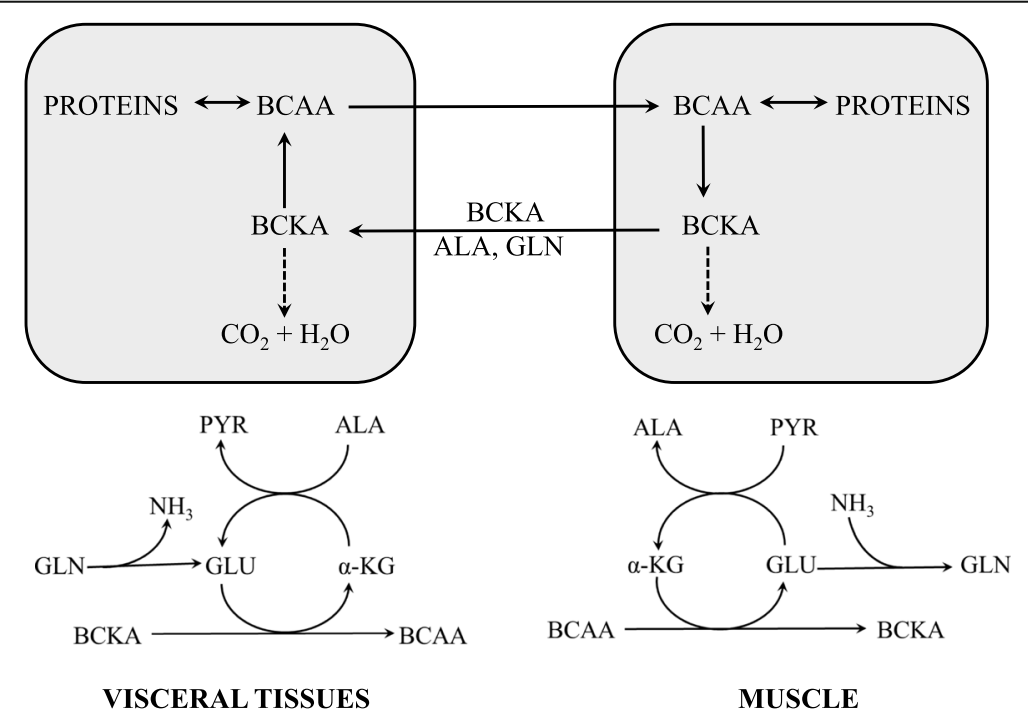

Fig. 3 The schemes of the BCAT reactions (BCAA deamination and BCKA amination) and supposed cycling of the BCAA and BCKA among organs, which may in various conditions attenuate the loss of essential BCAA [11]. ALA, alanine; BCAA, branched-chain amino acids; BCKA, branched-chain keto acids. GLU, glutamate; GLN, glutamine; PYR, pyruvate; a-KG, a-ketoglutarate 


\section{Functions of the BCAA}

The BCAAs serve as substrates for protein synthesis or energy production and perform several metabolic and signaling functions, particularly via activation of the mammalian target of rapamycin (mTOR) signaling pathway. The following roles of the BCAA should be considered as crucial for their use as nutritional supplements (Fig. 4).

\section{Effects on protein metabolism}

BCAAs not only serve as substrates for protein synthesis, but also exert stimulatory effect on protein synthesis and an inhibitory effect on proteolysis. The effects are realized by the BCAAs themselves, especially by leucine, and their metabolites. Leucine stimulates protein synthesis through the mTOR signaling pathway and phosphorylation of translation initiation factors and ribosomal proteins [12]. A role in protein anabolic effect of leucine plays also its stimulatory effect on insulin secretion [13]. The inhibitory effect of the BCAA on proteolysis is mediated mainly by BCKAs and HMB. BCKAs have been shown to prevent proteolysis in muscles under in vitro conditions [14]. Infusions of KIC were more effective than leucine in maintaining nitrogen balance in fasted subjects and in patients undergoing major abdominal surgery [15, 16]. HMB decreases the activity of the ubiquitin-proteasome proteolytic pathway and exerts beneficial effects on muscle in various conditions of health and disease [17].

\section{Effects on neurotransmission}

BCAAs are transported into the brain via the same carrier that transports aromatic amino acids (AAA; phenylalanine, tyrosine, tryptophan), and competition between BCAAs and AAAs may influence synthesis of some neurotransmitters, notably dopamine, norepinephrine, and 5-hydroxytryptamine (serotonin). Therefore, elevation of the BCAA in blood plasma is able to influence neurotransmitter levels in the brain with effects on behavior and brain function. This phenomenon is the rationale for use of the BCAAs in patients with liver cirrhosis, in which a decreased ratio of BCAAs to AAAs plays a role in pathogenesis of hepatic encephalopathy [18]. It is believed that BCAA supplementation attenuates production of serotonin, which is responsible for fatigue during exercise. Furthermore, BCAA transamination in the brain plays a role in the synthesis of glutamate and gamma-aminobutyric acid, and in ammonia detoxification to GLN in astrocytes. The studies have shown that leucine decreases appetite and may decrease body adiposity [19].

\section{Effects on glucose metabolism}

There are close associations between BCAAs and plasma glucose levels. The fact that BCAAs upregulate glucose transporters and activate insulin secretion has been widely demonstrated [13, 20, 21]. However, several researchers have suggested that excessive intake of amino acids could lead to inhibition of insulin signaling [22, 23]. Recent studies have suggested differential effects of each BCAA on glucose utilization and that BCAAs may induce insulin resistance through mTOR activation [24]. Further investigation is needed to understand variable reports ranging from improving glucose utilization to inducing insulin resistance.

\section{Effects mediated by ALA and GLN}

The rate of BCAA degradation in skeletal muscle is highly responsive to their availability [25]. The consequences of this phenomenon are that the primary effects of the consumption of a BCAA-enriched diet are activated catabolism of the BCAAs and enhanced levels of the

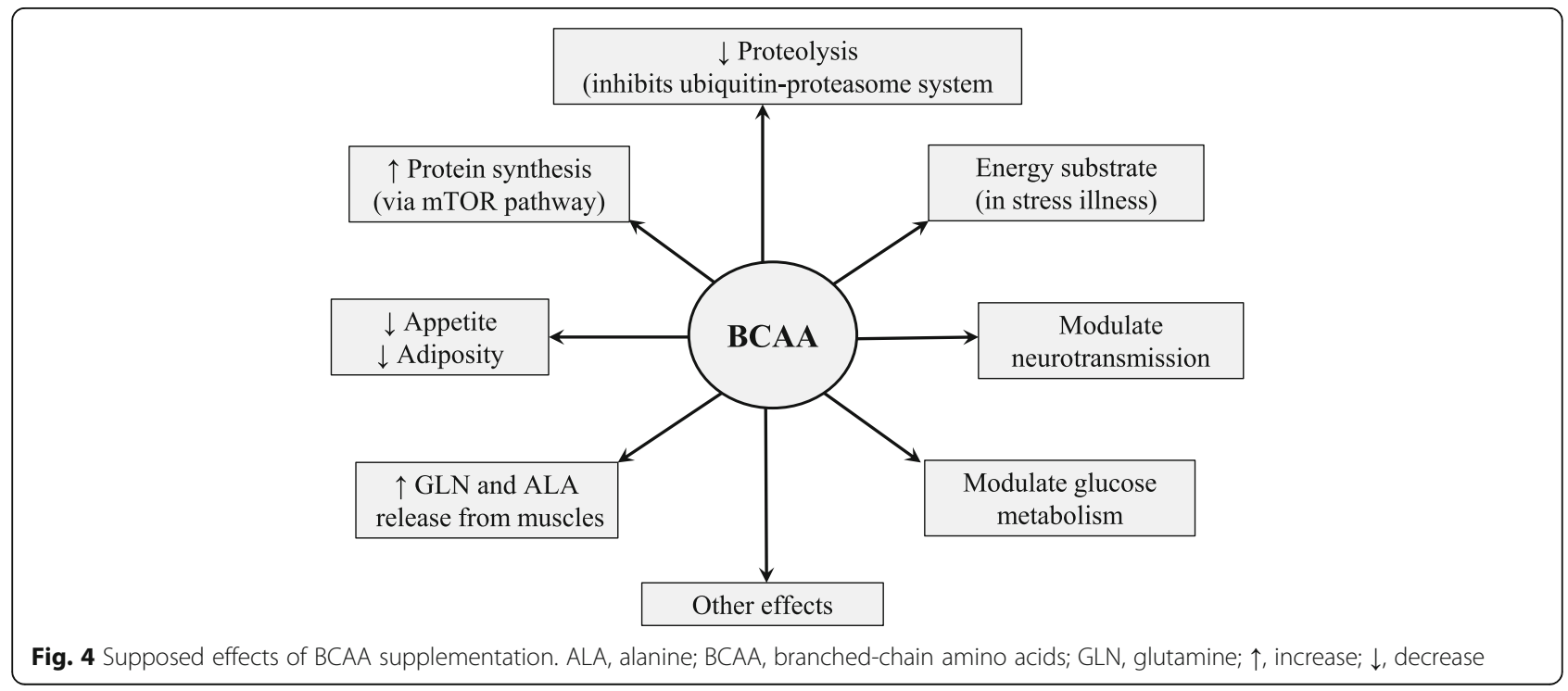


BCKAs, ALA, and GLN in peripheral circulation [26]. Therefore, a number of effects of BCAA supplementation are mediated by ALA and GLN. ALA is the main gluconeogenic amino acid, and GLN availability is essential for immune system, glutathione production, maintenance of acid-base balance by the kidneys, and expression of heat shock proteins.

\section{Other effects}

During recent years, a number of novel functions of BCAAs, including benefits for mammary health and milk quality, intestinal development, immune response, mitochondrial biogenesis and oxidative stress have been reported [21].

\section{Effects of starvation and diets with a different protein content on metabolism and BCAA levels}

BCAA metabolism is very sensitive to changes in the amount and composition of the food, which may occur in both healthy and disease states. Here, I have attempted to explain the effects of starvation and diets with low and high protein contents.

\section{Starvation}

Brief starvation uniquely increases BCAA concentrations in plasma. In humans, the increase is evident within a day, and reaches maximum by the second or third day [27, 28].

Both increased proteolysis and reduced protein synthesis in muscles have been reported during brief starvation and may explain the enhanced availability of BCAAs for muscles $[10,29,30]$. In this condition, BCAAs in muscles act as a source of nitrogen for synthesis of ALA and GLN, which are released into the blood and used in visceral tissues, especially as gluconeogenic substrates. Increased BCAT activity in muscles during starvation has been reported by several laboratories [31, 32].

Together with ALA and GLN, the BCKAs generated in $\mathrm{BCAT}$ reaction are released into circulation and their concentration in the blood increases [26]. It may be supposed that a portion of nitrogen released during catabolism of GLN and ALA in visceral tissues escapes utilization in the urea cycle and is used for amination of BCKAs. Higher rates of BCAA synthesis from the BCKAs were observed by the liver perfused with GLN-containing medium than that perfused with GLN-deficient medium [8]. A role in the increase of BCAAs may have also their decreased uptake from the blood due to the decreased levels of insulin. An unresolved possibility is the activated breakdown of proteins in the liver, which may, due to low activity of hepatic BCAT, result in the release of the BCAA into the blood.

Prolonged starvation lowers the BCAA concentration to basal levels and gradually increases the activity of the BCKD complex. Marked increase in BCKD activity in muscles and heart occurs in the terminal phase of starvation, when amino acids replace fatty acids and ketone bodies as the predominant energy substrate [33].

\section{Effects of a low-protein diet}

Feeding healthy human volunteers or animals a diet devoid of protein, but adequate in caloric content, lowered the plasma BCAA concentrations below basal levels [27, 34]. The amino acid pattern of children with severe kwashiorkor shows severe decrease of BCAAs [35].

It is believed that the principal factors in the decrease of BCAAs during protein deprivation are the absence of exogenous amino acids as well as curtailed muscle protein breakdown. Lowered BCKD activities in muscles and liver of protein-depleted rats indicate the effort of the body to conserve BCAAs [36].

BCAA or BCKA supplementation should be recommended when a low-protein diet is prescribed to patients with chronic renal failure or urea cycle disorders.

\section{Effects of a high-protein diet}

Increased intake of protein may increase protein synthesis, decrease protein breakdown, reduce fat accumulation, and increase fat-free mass. Therefore protein supplementation or a high-protein diet is recommended to build the muscles in athletes, to prevent muscle wasting in severe illness, and to lose fat in the treatment of obesity.

High concentrations of BCAAs and urea are found in the postprandial state in the peripheral blood and muscles after intake of a protein meal and in subjects consuming a high-protein diet. In contrast to increased BCAA levels, the increments in arterial concentrations of most remaining amino acids of the ingested protein are small or insignificant [37, 38].

The main cause of the specific BCAA increase is the unique distribution of the enzymes, which control BCAA catabolism. While complete oxidation of most individual amino acids occurs in the liver, the initial site of BCAA catabolism is skeletal muscle. Therefore, a significant portion of ingested BCAA escapes hepatic uptake and appears in peripheral circulation. The effects of protein ingestion on BCAA levels are not observed in a postabsorptive state [38].

\section{Disorders with decreased BCAA levels}

The studies have shown that BCAA deficiency impairs mRNA translation and dietary inadequacies of BCAA result in impaired growth and protein wasting $[12,39,40]$. In addition, studies in human subjects have shown that decreased BCAA level may influence synthesis of neurotransmitters and adversely affect brain function $[18,41]$. Therefore, BCAA supplementation seems rational in disorders with decreased BCAA levels, which occur in 
liver cirrhosis, urea cycle disorders, and chronic renal insufficiency.

\section{Liver cirrhosis}

The decrease in BCAAs and an increase in AAAs are characteristic alterations in the blood of subjects with liver cirrhosis, which play a role in pathogenesis of hepatic encephalopathy and muscle wasting [18, 42]. Several studies have shown an inverse relationship between plasma ammonia and BCAA concentrations in patients with cirrhosis and that ammonia infusion decreases BCAA levels $[43,44]$. BCAAs decrease because they are rapidly consumed to form glutamate from $\alpha-K G$ as a pivotal step in ammonia detoxification to GLN in muscles and in the brain [45]. Accelerated consumption of $\alpha-K G$ (cataplerosis) may disturb the function of the tricarboxylic acid (TCA) cycle (Fig. 5). The AAA increase is due to the decreased ability of the diseased liver to metabolize these amino acids. The BCAA levels do not decrease in acute liver injury due the leaking of amino acids from dying hepatocytes into the circulatory system [46].

\section{Effects of BCAA supplementation}

BCAAs are recommended to ameliorate cachexia and the decreased ratio of BCAAs to AAAs, which plays a role in the pathogenesis of hepatic encephalopathy. Potential benefits also include positive effects of the BCAA on ammonia detoxification to GLN in muscles, liver regeneration, albumin synthesis, immune and hepatic function, glucose metabolism, and physical and mental fatigue [20, 47-49].

Unfortunately, the results from clinical trials do not provide strong evidence of their beneficial effects $[50,51]$ and adverse effects of BCAA supplementation, which may compete with their benefits, have also been suggested [52]. The positive effects of BCAAs in subjects with liver cirrhosis may be blunted by enhanced catabolism of GLN produced in muscles to ammonia in visceral tissues, especially in the gut and kidneys. The draining of $\alpha-K G$ from TCA cycle may also be detrimental (Fig. 5). Therefore, therapeutic strategies are needed to avoid potential adverse effects of BCAAs on ammonia production and cataplerosis. Options include substitution of $\alpha-K G$, glutamate related substrates (e.g. L-ornithine-L-aspartate), GLN elimination from the body by phenylbutyrate, replacement of BCAAs by BCKAs, and optimizing dose, proportions, and timing of BCAA supplementation [52].

\section{Urea cycle disorders (UCD) and phenylbutyrate}

UCD result from inherited enzymatic defects in the ammonia detoxification pathway in the liver, leading to low levels of urea and high levels of ammonia in the blood. The disorders are characterized by seizures, lethargy, coma, and death in the neonatal period or severe longterm neurological impairment.

In addition to altered levels of ammonia and urea, common finding in patients with UCD is an increase in

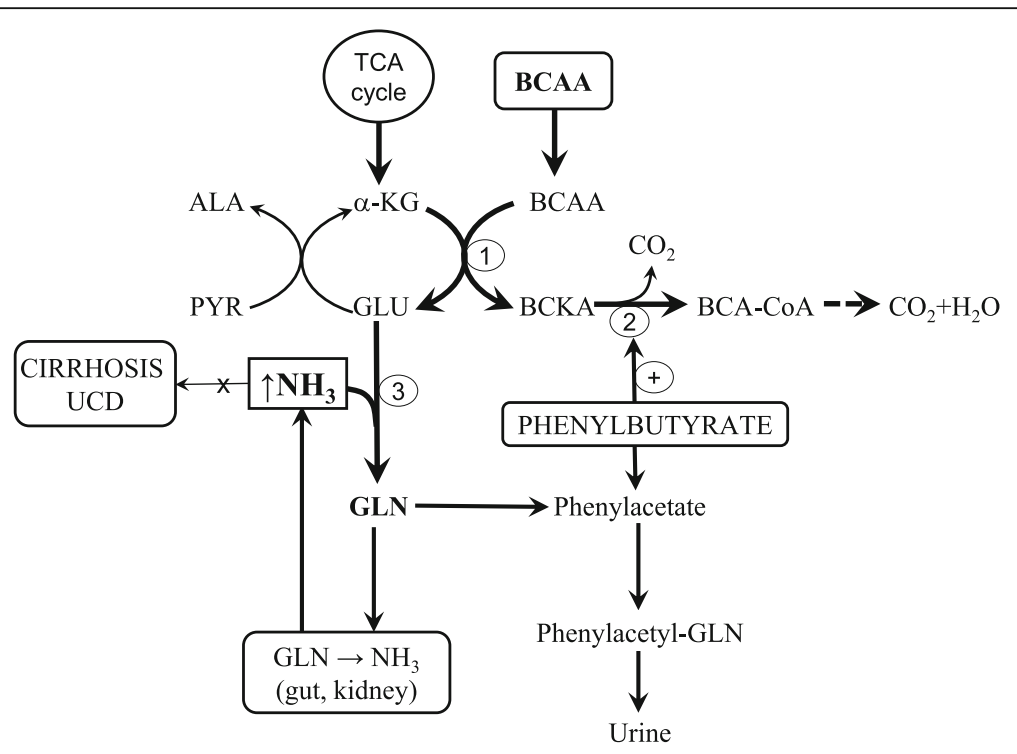

Fig. 5 Pathways of ammonia detoxification to GLN in muscles associated with enhanced consumption of the BCAA and a-KG (cataplerosis) and suggested effects of BCAA and phenylbutyrate in subjects with liver cirrhosis or UCD. Positive effect of BCAA on ammonia detoxification to GLN may be blunted by GLN degradation to ammonia in enterocytes and kidneys. Phenylbutyrate decreases ammonia via enhanced excretion of GLN by urine. An adverse side effect of phenylbutyrate is activation of BCKD resulting in the decrease of the BCAA. ALA, alanine; BCAA, branched-chain amino acids; BCKA, branched-chain keto acids. GLU, glutamate; GLN, glutamine; PYR, pyruvate; TCA cycle, tricarboxylic acid cycle; UCD, urea cycle disorders; a-KG, a-ketoglutarate. 1, branched-chain-amino-acid aminotransferase; 2, branched-chain a-keto acid dehydrogenase; 3, GLN synthetase 
GLN and a decline in BCAA levels, notably during acute metabolic decompensation [53]. These alterations support the theory that BCAAs play a unique role in ammonia detoxification to GLN and that hyperammonemia is the cause of decreased BCAA levels in subject with liver cirrhosis $[45,54]$.

At present, the management of UCD is achieved by dietary protein restriction and the use of compounds that remove nitrogen, notably benzoate and phenylbutyrate. Benzoate conjugates glycine to promote the synthesis of hippuric acid that is eliminated in urine and thus attenuates catabolism of glycine to ammonia. Phenylbutyrate is converted by $\beta$-oxidation into phenylacetate that is conjugated with GLN to form phenacetylglutamine, which is excreted in the urine (Fig. 4). Unfortunately, it has been shown that phenylbutyrate activates the BCKD, resulting in decreases in BCAA and BCKA levels in blood plasma $[55,56]$. Marked decrease of BCAAs in UCD after phenylbutyrate treatment has been reported by Scaglia et al. [57].

\section{Effects of BCAA supplementation}

Low BCAA levels in subjects with UCD, especially those treated by phenylbutyrate, indicate the rationale to use BCAAs as a therapeutic agent. Unfortunately, the reports of attempts to use BCAAs in UCD are unique. Cross-sectional data from 41 European Inherited Metabolic Disorder centers reported that only $16(3 \%)$ patients (from 8 centers in 5 countries) received BCAA supplements. The two most common conditions were ornithine transcarbamylase deficiency and citrullinaemia [58].

\section{Chronic renal failure (CRF)}

Most studies of amino acid patterns in CRF reported decreased BCAA and BCKA levels in the blood plasma [59-61] and reduced concentrations of valine in muscles $[61,62]$. The derangements are caused by the action of multiple factors, notably acidosis and glucocorticoids. Decreased intake of proteins and hemodialysis, resulting in low concentrations of most essential and nonessential amino acids, is also a factor. In contrast to CRF, inconsistent alterations have been reported in acute renal failure.

Several articles have suggested that metabolic acidosis is responsible for accelerated proteolysis and enhanced activity of the BCKD in muscles and liver [63, 64]. More significant increases in proteolysis and leucine oxidation were reported in rats with chronic uremia and acidosis when compared with uremic rats without acidosis. A significant decrease in valine concentration in the gastrocnemius muscle was found only in rats with acidosis [61].

\section{Effects of BCAA supplementation}

BCAAs and BCKAs are supplied to patients with CRF together with other essential amino acids and their ketoanalogues to decrease protein intake as much as possible to maintain protein balance and avoid its deleterious effects on urea levels $[65,66]$.

\section{Disorders with enhanced BCAA levels}

Increased BCAA concentrations are found in various insulin-deficient and -resistant states, especially diabetes and obesity. Very high BCAA and BCKA concentrations are found in maple syrup urine disease (MSUD).

\section{Type 1 diabetes}

High BCAA levels in subjects with defective insulin secretion were first described in dogs with experimental diabetes [67]. Further studies have shown that in addition to the increase of BCAAs, there is a decrease in levels of gluconeogenic amino acids, especially ALA [68-70]. Most data on pathogenesis of high levels of the BCAA in diabetes type 1 originate from studies using animals with diabetes induced by streptozotocin or alloxan.

There are some similarities in the pathogenesis of the increased BCAAs in diabetes and short-term starvation, which is also an insulin deficient state. As in starvation, a role play activated amination of the BCKAs in the liver and impaired uptake of the BCAA by muscles. The BCKA levels increase in blood plasma and muscles of rats with chemically-induced diabetes, but decline in the liver [71]. The role of the liver as a source of BCAAs is supported by observations of reduced activity of hepatic BCKD in rats with severe ketotic diabetes [72].

However, unlike brief starvation, the changes in diabetes are associated with marked increase in proteolysis and BCKD activity in muscles, resulting in severe cachexia [73]. While muscle nitrogen repletion occurs and BCAA levels are normalized after feeding of previously starving subjects, the BCAAs accumulate and diminished nitrogen repletion remains after feeding in subjects with type 1 diabetes [74].

\section{Obesity and type 2 diabetes}

Plasma concentrations of BCAAs are frequently elevated in obesity and type 2 diabetes [75-77]. The mechanism responsible for the increased BCAAs in these insulinresistant states is not completely clear. A major cause might be reduced activity of the BCKA dehydrogenase, which was reported in the liver and adipose tissue in genetically obese ob/ob mice, Zucker rats and spontaneous type 2 diabetes Otsuka Long-Evans Tokushima Fatty (OLETF) rats [76, 77].

The studies have shown that the BCAA levels in obesity correlate with insulin resistance and are a sensitive predictor of diabetes in the future $[78,79]$. Recent studies have suggested that high levels of the BCAA interfere with oxidation of fatty acids in muscles, leading to accumulation of various acylcarnitines and insulin resistance [24]. 


\section{$B C A A$ supplementation}

Conflicting results have been reported concerning the effects of BCAA supplementation in subjects with insulin resistance. Leucine improved glucose tolerance, decreased hepatic steatosis, and decreased inflammation in adipose tissue in mice fad a high-fat diet [80] and rescued insulin signaling in adipose tissue obtained from insulin resistant $\mathrm{db} / \mathrm{db}$ mice [81]. Arakawa et al. [82] reported that BCAAs reduced hepatic and triglyceride concentrations in mice fed a high-fat diet. On the other hand, Newgard et al. [79] showed that administration of a mixture of BCAA to rats on a high-fat diet increased insulin resistance. White et al. [24] demonstrated that the BCAA-restricted diet improved muscle insulin sensitivity in Zucker-fatty rats.

\section{Maple syrup urine disease (MSUD)}

MSUD is recessive disorder caused by a severe deficiency of BCKD activity. All three BCAAs, as well as the corresponding BCKAs, are elevated in blood, tissues, and urine. High BCAA and BCKA levels are related to excitotoxicity, energy deficit, and oxidative stress in the brain, resulting in severe neurological symptoms.

\section{$B C A A$ supplementation}

BCAA administration to subjects with MSUD is inappropriate. DNA damage in the hippocampus and the striatum was demonstrated after administration of BCAAs in an animal model of MSUD [83]. Current treatment of MSUD is based on protein restriction and synthetic formulas with reduced BCAA content. Perspective may be phenylbutyrate, which activates $\mathrm{BCKD}$ and decreases $\mathrm{BCAA}$ and BCKA levels [55, 56]. Unfortunately, studies examining phenylbutyrate in MSUD patients are unique. Decreased BCAA and BCKA levels were reported in three out of the five MSUD patients treated by phenylbutyrate $\left(10 \mathrm{~g} / \mathrm{m}^{2}\right)$ for one day [56]. Long-term studies in different MSUD phenotypes are indicated to verify phenylbutyrate efficacy.

\section{Conditions with enhanced BCAA catabolism and inconsistent alterations in BCAA levels \\ Exercise}

Physical exercise is associated with enhanced BCAA oxidation and GLN release from muscles [84, 85]. Evidence suggests that BCKD is activated by dephosphorylation mediated by falling ATP levels within the muscles during exercise. Training appears to increase mRNA expression of this enzyme [86]. The plasma BCAA levels during or after exercise have been reported to be unchanged [87], to decrease [88], or to increase [89]. The cause of inconsistent response can be explained by different work load and duration of exercise.

\section{Effects of BCAA supplementation}

BCAAs are recognized as supplements for athletes with a number of benefits, notably on muscle protein synthesis, fatigue recovery, and exercise-induced muscle damage [90]. In addition to the positive reports, there are a number of reports showing no benefits of BCAA supplementation [91]. Of special interest should be findings of enhanced blood ammonia levels after BCAA administration during exercise suggesting that exogenous $\mathrm{BCAA}$ may exert negative effects on muscle performance via ammonia $[92,93]$. Additional studies are needed to assess the true efficacy of BCAA supplementation on muscle performance and fatigue.

\section{Hypermetabolic states accompanied by systemic inflammatory response syndrome}

There are several hypermetabolic states (e.g. sepsis, burn injury, trauma, and cancer) in which alterations in BCAA levels are not consistent, with increased, unchanged, and decreased levels being reported. Present in all of these conditions is systemic inflammatory response syndrome (SIRS) characterized by a wide range of neuro-humoral abnormalities, including enhanced production of cytokines, sympathetic nervous system activation, and cortisol production. These events cause several alterations in metabolism, including insulin resistance and enhanced myofibrillar protein degradation, resulting in severe depletion of lean body mass. If the hypermetabolic state persists, multisystem organ failure and eventually death may occur (Fig. 6).

In this situation, BCAAs act as a significant energy substrate for muscles $[4,5,94]$. Increased BCAA oxidation is coupled with increased synthesis of GLN, which is released from muscles and utilized, preferably by the immune system. Utilization of GLN often exceeds its synthesis, leading to a lack of GLN in blood and tissues $[95,96]$. Decreased GLN availability can become ratelimiting for key functions of immune cells, such as phagocytosis and antibody production. Decreased GLN levels have been shown to act as a driving force for BCAA utilization in muscles [97]. Studies have also indicated that inflammatory signals decrease BCAA absorption from the gut and inhibit BCAA transport from the blood to muscles, while promoting transport into the liver $[98,99]$. The BCAA synthesis from the BCKA in visceral tissues is probably activated. A marked increase in leucine release was observed by the isolated liver of endotoxin-treated animals after the addition of KIC into perfusion medium [7].

The cause of inconsistent alterations in BCAA levels although their oxidation is remarkably activated are different influences of individual metabolic changes occurring in the SIRS. Increased protein breakdown or decreased protein synthesis in muscles and insulin resistance may enhance the 


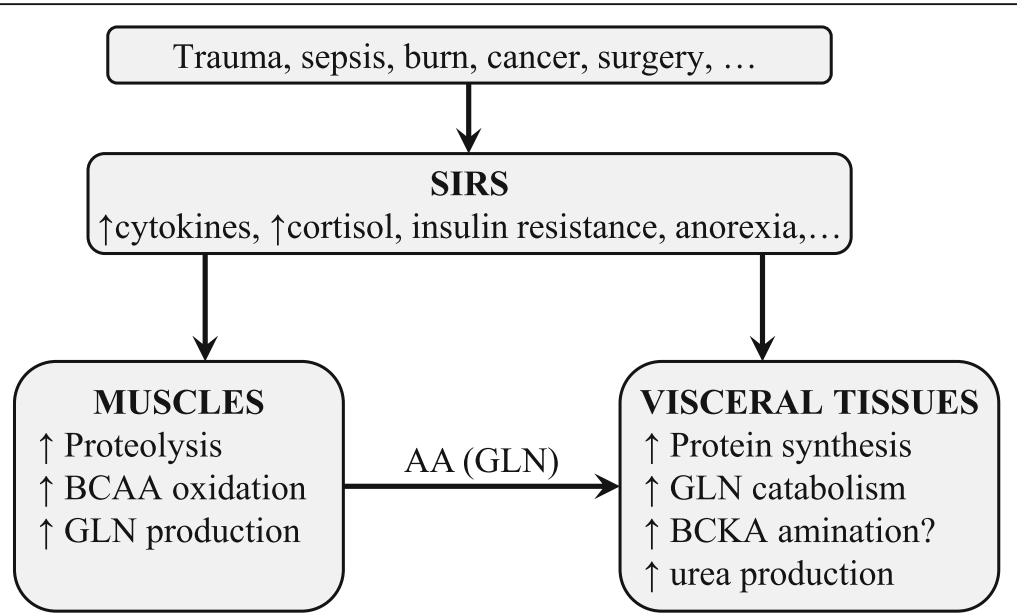

Fig. 6 Main alterations in protein and BCAA metabolism in disorders accompanied by SIRS. AA, amino acids; BCAA, branched-chain amino acids; BCKA, branched-chain keto acids; GLN, glutamine; SIRS, systemic inflammatory response syndrome; $\uparrow$, increase; $\downarrow$, decrease

BCAA levels. Activation of BCAA catabolism associated with enhanced ALA and GLN production in muscles and protein synthesis in visceral tissues decrease the BCAA levels. Therefore, alterations in BCAA levels are inconsistent.

\section{BCAA supplementation in burn, trauma, and sepsis}

Rationales for the use of BCAA supplements in conditions with SIRS are their enhanced oxidation, which may limit their availability in tissues and their protein anabolic properties. Benefits of BCAAs may also be related to their role as a precursor of GLN, which is a key factor in maintaining immune functions and gut integrity, and has a favorable influence on protein balance.

Various solutions containing different amounts and proportions of individual BCAA have been used to examine their effects in trauma, burn, or sepsis. A number of investigators have reported that BCAA ameliorate negative nitrogen balance [100-102]. However, the results of other investigators have not been impressive, and there is no scientific consensus regarding the effect BCAA-enriched formulas on protein balance, length of hospital stay, and mortality [103-105]. A serious shortcoming of most of the studies is the lack of information regarding BCAA concentrations in blood and tissues, which may be suggested as a possible criterion of eligibility of the indication.

The low effectiveness of the BCAA in disorders with the presence of SIRS may be related to insulin resistance and metabolic alteration associated with inflammation. Studies have shown that inflammatory response blunts the anabolic response to BCAA administration. Lang and Frost [106] demonstrated that leucine induced activation of eukaryotic initiation factor eIF4E is abrogated in endotoxintreated rats and that endotoxin treatment antagonized the leucine-induced phosphorylation of ribosomal protein S6 and mTOR.

In recent years articles have emerged suggesting positive effects of BCAA in traumatic brain injury. In rodents, BCAAs have demonstrated to ameliorate injury-induced cognitive impairment [107], and clinical studies have demonstrated that BCAAs enhance the cognitive recovery in patients with severe traumatic brain injury $[108,109]$.

\section{$B C A A$ supplementation in cancer}

Unlike other states accompanied by SIRS, muscle wasting and amino acid mobilization from muscles in subjects with cancer may be driven by secretion of different tumor-derived mediators. Therefore, progressive depletion of muscle mass may be observed in some cancer patients. Also high rates of BCAA oxidation in muscles of subjects with cancer have been reported [110]. Increasing evidence demonstrates that BCAAs are essential nutrients for cancer growth and are used as a source of energy by tumors. Expression of the cytosolic type of BCAT has been shown to correlate with more aggressive cancer growth [111].

The findings of clinical trials examining the effects of BCAA-enriched nutritional support to cancer patients are inconsistent. Some showed improved nitrogen balance and reduced skeletal muscle catabolism whereas others show no significant improvement [112]. A concern in the tumor-bearing state is that provision of the BCAA will promote tumor growth.

\section{Summary and conclusions}

The studies indicate that important role in pathogenesis of alterations in BCAA metabolism play: (i) skeletal muscle as initial site of BCAA catabolism accompanied by the release of GLN, ALA, and BCKA to the blood; (ii) activity of BCKD in muscles and liver, and (iii) amination 
of BCKA to corresponding BCAA, especially by nitrogen of ALA and GLN released from muscles. Here are examples of importance of these metabolic steps:

ad (i) Because the muscle is the initial site of BCAA catabolism, marked rise of BCAA is observed after a meal while the rise of other amino acids is small. Enhanced consumption of the BCAA for ammonia detoxification to GLN in muscles is the main cause of the decrease of the BCAA in hyperammonemic conditions (liver cirrhosis, UCD). Increased production of GLN after BCAA intake in muscles may lead to enhanced production of ammonia in enterocytes and kidneys with deleterious effect in subjects with liver disease.

ad (ii) Decreased BCKD activity is the main cause of increased BCAA and BCKA levels in MSUD and may play a role in increased BCAA levels in obesity and type 2 diabetes. Increased BCKD activity is responsible for the decrease of BCAAs in CRF and enhanced oxidation of BCAAs during exercise and in various hypermetabolic conditions (burn, sepsis, trauma, cancer).

ad (iii) BCKA amination partially explains the increased BCAA concentrations during brief starvation and in type 1 diabetes, and is the basis of rationale to use BCKA-enriched supplements in CRF therapy.

Although amino acid concentrations in the plasma pool are poor indicators of their requirements, it may be suggested that under conditions of good understanding of the BCAA metabolism in specific disorder, the BCAA levels would conceptually be an acceptable argument for their supplementation. It may be supposed that:

1. Together with requirements to decrease protein content in a diet, increased oxidation and low BCAA levels are a clear rationale to use the BCAA together with other essential amino acids and their ketoanalogues in CRF therapy.

2. Although BCAA decrease in blood plasma is a rationale to use the BCAA supplements in patients with liver cirrhosis and UCD, therapeutic strategies are needed to avoid detrimental effects of BCAA supplementation on ammonia production.

3. Further studies are necessary to conclude the question of the effects of BCAA supplementation in burn, trauma, sepsis, cancer, and exercise. A very small number of clinical studies have reported the effects of BCAA supplementation in relation to amino acid concentrations in blood and tissues.

4. Whether increased BCAA levels only markers are or also contribute to insulin resistance should be known before the decision is taken regarding their suitability in obese subjects and patients with type 2 diabetes.
In conclusion, alterations in BCAA metabolism are common in a number of disease states and the BCAA have therapeutic potential due to their proven protein anabolic effects. However, many controversies about the use of BCAAs in clinical practice still exist, and careful studies are needed to elucidate the effectiveness of BCAAs in most indications.

\section{Abbreviations \\ KIC: a-ketoisocaproate (ketoleucine); KIV: a-ketoisovalerate (ketovaline); KMV: a-keto- $\beta$-methylvalerate (ketoisoleucine); ALA: Alanine; \\ BCAA: Branched-chain amino acids; BCAT: Branched-chain-amino-acid aminotransferase; BCKA: Branched-chain keto acids; BCKD: Branched-chain a-keto acid dehydrogenase; CRF: Chronic renal failure; GLN: Glutamine; MSUD: Maple syrup urine disease; SIRS: Systemic inflammatory response syndrome; TCA cycle: Tricarboxylic acid cycle; UCD: Urea cycle disorders; a-KG, a-ketoglutarate}

Funding

The author was supported by the program PROGRES Q40/02.

Authors' contributions

$\mathrm{MH}$ performed the literature search and wrote the manuscript. The author read and approved the final manuscript.

Ethics approval and consent to participate

Not applicable.

\section{Competing interests}

The author declares that he/she has no competing interests.

\section{Publisher's Note}

Springer Nature remains neutral with regard to jurisdictional claims in published maps and institutional affiliations.

Received: 7 March 2018 Accepted: 18 April 2018

Published online: 03 May 2018

\section{References}

1. Chen L, Chen Y, Wang X, Li H, Zhang H, Gong J, Shen S, Yin W, Hu H. Efficacy and safety of oral branched-chain amino acid supplementation in patients undergoing interventions for hepatocellular carcinoma: a metaanalysis. Nutr J. 2015;14:67.

2. Bifari $F$, Nisoli E. Branched-chain amino acids differently modulate catabolic and anabolic states in mammals: a pharmacological point of view. $\mathrm{Br} J$ Pharmacol. 2017:174:1366-77.

3. Harper AE, Miller RH, Block KP. Branched-chain amino acid metabolism. Annu Rev Nutr. 1984:4:409-54.

4. Holecek M. Leucine metabolism in fasted and tumor necrosis factor-treated rats. Clin Nutr. 1996:15:91-3.

5. Holecek M, Sprongl L, Skopec F, Andrýs C, Pecka M. Leucine metabolism in TNF-a- and endotoxin-treated rats: contribution of hepatic tissue Am J Phys 1997;273: E1052-E1058.

6. Swain LM, Shiota T, Walser M. Utilization for protein synthesis of leucine and valine compared with their keto analogues. Am J Clin Nutr. 1990;51:411-5.

7. Holeček M, Šprongl L, Tichý M, Pecka M. Leucine metabolism in rat liver after a bolus injection of endotoxin. Metabolism. 1998;47:681-5.

8. Holecek M, Rysava R, Safranek R, Kadlcikova J, Sprongl L. Acute effects of decreased glutamine supply on protein and amino acid metabolism in hepatic tissue: a study using isolated perfused rat liver. Metabolism. 2003;52:1062-7.

9. Adibi SA. Influence of dietary deprivations on plasma concentration of free amino acids of man. J Appl Physiol. 1968;25:52-7.

10. Holeček M, Mičuda S. Amino acid concentrations and protein metabolism of two types of rat skeletal muscle in postprandial state and after brief starvation. Physiol Res. 2017;66:959-67.

11. Holecek M. The BCAA-BCKA cycle: its relation to alanine and glutamine synthesis and protein balance. Nutrition. 2001;17:70.

12. Nair KS, Short KR. Hormonal and signaling role of branched-chain amino acids. J Nutr. 2005;135:1547S-52S. 
13. Floyd JC Jr, Fajans SS, Conn JW, Knopf RF, Rull J. Stimulation of insulin secretion by amino acids. J Clin Invest. 1966;45:1487-502.

14. Tischler ME, Desautels M, Goldberg AL. Does leucine, leucyl-tRNA, or some metabolite of leucine regulate protein synthesis and degradation in skeletal and cardiac muscle? J Biol Chem. 1982;257:1613-21.

15. Mitch WE, Walser M, Sapir DG. Nitrogen sparing induced by leucine compared with that induced by its keto analogue, alpha-ketoisocaproate, in fasting obese man. J Clin Invest. 1981;67:553-62.

16. Sapir DG, Stewart PM, Walser M, Moreadith C, Moyer ED, Imbembo AL, et al Effects of alpha-ketoisocaproate and of leucine on nitrogen metabolism in postoperative patients. Lancet. 1983;1(8332):1010-4.

17. Holeček M. Beta-hydroxy-beta-methylbutyrate supplementation and skeletal muscle in healthy and muscle-wasting conditions. J Cachexia Sarcopenia Muscle. 2017:8:529-41.

18. Fischer JE, Funovics JM, Aguirre A, James JH, Keane JM, Wesdorp Rl, et al. The role of plasma amino acids in hepatic encephalopathy. Surgery. 1975; 78:276-90.

19. Pedroso JA, Zampieri TT, Donato J. Reviewing the effects of L-leucine supplementation in the regulation of food intake, energy balance, and glucose homeostasis. Nutrients. 2015;7:3914-37.

20. Nishitani S, Takehana K, Fujitani S, Sonaka I. Branched-chain amino acids improve glucose metabolism in rats with liver cirrhosis. Am J Physiol Gastrointest Liver Physiol. 2005;288:G1292-300.

21. Zhang S, Zeng X, Ren M, Mao X, Qiao S. Novel metabolic and physiological functions of branched chain amino acids: a review. J Anim Sci Biotechnol. 2017:8:10.

22. Um SH, D'Alessio D, Thomas G. Nutrient overload, insulin resistance, and ribosomal protein S6 kinase 1, S6K1. Cell Metab. 2006;3:393-402.

23. Tremblay F, Lavigne $C$, Jacques $H$, Marette A. Role of dietary proteins and amino acids in the pathogenesis of insulin resistance. Annu Rev Nutr. 2007; 27:293-310

24. White PJ, Lapworth AL, An J, Wang L, McGarrah RW, Stevens RD, et al. Branched-chain amino acid restriction in Zucker-fatty rats improves muscle insulin sensitivity by enhancing efficiency of fatty acid oxidation and acylglycine export. Mol Metab. 2016;5:538-51.

25. Manchester KL. Oxidation of amino acids by isolated rat diaphragm and the influence of insulin. Biochim Biophys Acta. 1965;100:295-8.

26. Holecek M, Siman P, Vodenicarovova M, Kandar R. Alterations in protein and amino acid metabolism in rats fed a branched-chain amino acid- or leucine-enriched diet during postprandial and postabsorptive states. Nutr Metab (Lond). 2016;13:12.

27. Adibi SA. Metabolism of branched-chain amino acids in altered nutrition. Metabolism. 1976:25:1287-302.

28. Schauder P, Herbertz L, Langenbeck U. Serum branched chain amino and keto acid response to fasting in humans. Metabolism. 1985;34:58-61.

29. Fryburg DA, Barrett EJ, Louard RJ, Gelfand RA. Effect of starvation on human muscle protein metabolism and its response to insulin. Am J Phys. 1990;259: E477-82.

30. Holecek M, Sprongl L, Tilser I. Metabolism of branched-chain amino acids in starved rats: the role of hepatic tissue. Physiol Res. 2001;50:25-33.

31. Adibi SA, Peterson JA, Krzysik BA. Modulation of leucine transaminase activity by dietary means. Am J Phys. 1975;228:432-5.

32. Sketcher RD, Fern EB, James WP. The adaptation in muscle oxidation of leucine to dietary protein and energy intake. Br J Nutr. 1974;31:333-42.

33. Holecek M. Effect of starvation on branched-chain alpha-keto acid dehydrogenase activity in rat heart and skeletal muscle. Physiol Res. 2001; 50:19-24.

34. Grimble RF, Whitehead RG. Changes in the concentration of specific amino acids in the serum of experimentally malnourished pigs. Br J Nutr. 1970;24:557-64.

35. Holt LE, Snyderman SE, Norton PM, Roitman E, Finch J. The plasma aminogram in kwashiorkor. Lancet. 1963;2(7322):1342-8.

36. Reeds PJ. The catabolism of valine in the malnourished rat. Studies in vivo and in vitro with different labelled forms of valine. Br J Nutr. 1974;31:259-70.

37. Wahren J, Felig P, Hagenfeldt L. Effect of protein ingestion on splanchnic and leg metabolism in normal man and in patients with diabetes mellitus. J Clin Invest. 1976:57:987-99.

38. Holecek M, Kovarik M. Alterations in protein metabolism and amino acid concentrations in rats fed by a high-protein (casein-enriched) diet - effect of starvation. Food Chem Toxicol. 2011;49:3336-42.

39. Watford M. Lowered concentrations of branched-chain amino acids result in impaired growth and neurological problems: insights from a branched- chain alpha-keto acid dehydrogenase complex kinase-deficient mouse model. Nutr Rev. 2007:65:167-72.

40. Anthony TG, Reiter AK, Anthony JC, Kimball SR, Jefferson LS. Deficiency of dietary EAA preferentially inhibits mRNA translation of ribosomal proteins in liver of meal-fed rats. Am J Physiol Endocrinol Metab. 2001;281:E430-9.

41. Blomstrand E. Amino acids and central fatigue. Amino Acids. 2001;20:25-34

42. Dasarathy S, Hatzoglou M. Hyperammonemia and proteostasis in cirrhosis. Curr Opin Clin Nutr Metab Care. 2018:21:30-6.

43. Leweling H, Breitkreutz R, Behne F, Staedt U, Striebel JP, Holm E. Hyperammonemia-induced depletion of glutamate and branched-chain amino acids in muscle and plasma. J Hepatol. 1996;25:756-62.

44. Holeček M, Šprongl L, Tichý M. Effect of hyperammonemia on leucine and protein metabolism in rats. Metabolism. 2000;49:1330-4.

45. Holecek M, Kandar R, Sispera L, Kovarik M. Acute hyperammonemia activates branched-chain amino acid catabolism and decreases their extracellular concentrations: different sensitivity of red and white muscle. Amino Acids. 2011:40:575-84.

46. Holeček M, Mráz J, Tilšer I. Plasma amino acids in four models of experimental liver injury in rats. Amino Acids. 1996;10:229-41.

47. Davis JM, Alderson NL, Welsh RS. Serotonin and central nervous system fatique: nutritional considerations. Am J Clin Nutr. 2000;72:573S-8S.

48. Holecek M. Three targets of branched-chain amino acid supplementation in the treatment of liver disease. Nutrition. 2010;26:482-90.

49. Holecek M, Simek J, Palicka V, Zadák Z. Effect of glucose and branched chain amino acid (BCAA) infusion on onset of liver regeneration and plasma amino acid pattern in partially hepatectomized rats. J Hepatol. 1991;13:14-20.

50. Als-Nielsen B, Koretz RL, Kjaergard LL, Gluud C. Branched-chain amino acids for hepatic encephalopathy. Cochrane Database Syst Rev. 2003;2:CD001939.

51. Gluud LL, Dam G, Les I, Córdoba J, Marchesini G, Borre M, et al. Branchedchain amino acids for people with hepatic encephalopathy. Cochrane Database Syst Rev. 2015;9:CD001939.

52. Holeček M. Branched-chain amino acid supplementation in treatment of liver cirrhosis: updated views on how to attenuate their harmful effects on cataplerosis and ammonia formation. Nutrition. 2017:41:80-5.

53. Rodney S, Boneh A. Amino acid profiles in patients with urea cycle disorders at admission to hospital due to metabolic decompensation. JIMD Rep. 2013;9:97-104.

54. Holecek M. Evidence of a vicious cycle in glutamine synthesis and breakdown in pathogenesis of hepatic encephalopathy-therapeutic perspectives. Metab Brain Dis. 2014;29:9-17.

55. Holecek M, Vodenicarovova M, Siman P. Acute effects of phenylbutyrate on glutamine, branched-chain amino acid and protein metabolism in skeletal muscles of rats. Int J Exp Pathol. 2017;98:127-33.

56. Brunetti-Pierri N, Lanpher B, Erez A, Ananieva EA, Islam M, Marini JC, et al. Phenylbutyrate therapy for maple syrup urine disease. Hum Mol Genet. 2011;20:631-40

57. Scaglia F, Carter S, O'Brien WE, Lee B. Effect of alternative pathway therapy on branched chain amino acid metabolism in urea cycle disorder patients. Mol Genet Metab. 2004;81:S79-85.

58. Adam S, Almeida MF, Assoun M, Baruteau J, Bernabei SM, Bigot S, et al. Dietary management of urea cycle disorders: European practice. Mol Genet Metab. 2013;110:439-45

59. Schauder P, Matthaei D, Henning HV, Scheler F, Langenbeck U. Blood levels of branched-chain amino acids and alpha-ketoacids in uremic patients given keto analogues of essential amino acids. Am J Clin Nutr. 1980;33:1660-6.

60. Garibotto G, Paoletti E, Fiorini F, Russo R, Robaudo C, Deferrari G, Tizianello A. Peripheral metabolism of branched-chain keto acids in patients with chronic renal failure. Miner Electrolyte Metab. 1993;19:25-31.

61. Holecek M, Sprongl L, Tilser I, Tichý M. Leucine and protein metabolism in rats with chronic renal insufficiency. Exp Toxicol Pathol. 2001;53:71-6.

62. Alvestrand $A$, Fürst $P$, Bergström J. Plasma and muscle free amino acids in uremia: influence of nutrition with amino acids. Clin Nephrol. 1982;18:297-305.

63. Hara Y, May RC, Kelly RA, Mitch WE. Acidosis, not azotemia, stimulates branched-chain, amino acid catabolism in uremic rats. Kidney Int. 1987;32: 808-14

64. May RC, Masud T, Logue B, Bailey J, England BK. Metabolic acidosis accelerates whole body protein degradation and leucine oxidation by a glucocorticoiddependent mechanism. Miner Electrolyte Metab. 1992;18:245-9.

65. Teplan V, Schück O, Horácková M, Skibová J, Holecek M. Effect of a keto acid-amino acid supplement on the metabolism and renal elimination of branched-chain amino acids in patients with chronic renal insufficiency on a low protein diet. Wien Klin Wochenschr. 2000;112:876-81. 
66. Kovesdy CP, Kopple JD, Kalantar-Zadeh K. Management of protein-energy wasting in non-dialysis-dependent chronic kidney disease: reconciling low protein intake with nutritional therapy. Am J Clin Nutr. 2013;97:1163-77.

67. Ivy JH, Svec M, Freeman S. Free plasma levels and urinary excretion of eighteen amino acids in normal and diabetic dogs. Am J Phys. 1951;167:182-92.

68. Borghi L, Lugari R, Montanari A, Dall'Argine P, Elia GF, Nicolotti V, et al. Plasma and skeletal muscle free amino acids in type I, insulin-treated diabetic subjects. Diabetes. 1985;34:812-5.

69. Rodríguez T, Alvarez B, Busquets S, Carbó N, López-Soriano FJ, Argilés JM. The increased skeletal muscle protein turnover of the streptozotocin diabetic rat is associated with high concentrations of branched-chain amino acids. Biochem Mol Med. 1997;61:87-94.

70. Jensen-Waern M, Andersson M, Kruse R, Nilsson B, Larsson R, Korsgren O, Essén-Gustavsson B. Effects of streptozotocin-induced diabetes in domestic pigs with focus on the amino acid metabolism. Lab Anim. 2009;43:249-54.

71. Hutson SM, Harper AE. Blood and tissue branched-chain amino and alphaketo acid concentrations: effect of diet, starvation, and disease. Am J Clin Nutr. 1981;34:173-83.

72. Gibson R, Zhao Y, Jaskiewicz J, Fineberg SE, Harris RA. Effects of diabetes on the activity and content of the branched-chain alpha-ketoacid dehydrogenase complex in liver. Arch Biochem Biophys. 1993;306:22-8.

73. Aftring RP, Miller WJ, Buse MG. Effects of diabetes and starvation on skeletal muscle branched-chain alpha-keto acid dehydrogenase activity. Am J Phys. 1988;254:E292-300.

74. Felig P, Wahren J, Sherwin R, Palaiologos G. Amino acid and protein metabolism in diabetes mellitus. Arch Intern Med. 1977;137:507-13.

75. Carlsten A, Hallgren B, Jagenburg R, Svanborg A, Werkö L. Amino acids and free fatty acids in plasma in diabetes. I. The effect of insulin on the arterial levels. Acta Med Scand. 1966;179:361-70.

76. She P, Van Horn C, Reid T, Hutson SM, Cooney RN, Lynch CJ. Obesity-related elevations in plasma leucine are associated with alterations in enzymes involved in branched-chain amino acid metabolism. Am J Physiol Endocrinol Metab. 2007;293:E1552-63.

77. Kuzuya T, Katano Y, Nakano I, Hirooka Y, Itoh A, Ishigami M, et al. Regulation of branched-chain amino acid catabolism in rat models for spontaneous type 2 diabetes mellitus. Biochem Biophys Res Commun. 2008;373:94-8.

78. Wang TJ, Larson MG, Vasan RS, Cheng S, Rhee EP, McCabe E, et al. Metabolite profiles and the risk of developing diabetes. Nat Med. 2011;17:448-53.

79. Newgard CB, An J, Bain JR, Muehlbauer MJ, Stevens RD, Lien LF, et al. A branched-chain amino acid-related metabolic signature that differentiates obese and lean humans and contributes to insulin resistance. Cell Metab. 2009:9:311-26.

80. Macotela Y, Emanuelli B, Bång AM, Espinoza DO, Boucher J, Beebe K, et al. Dietary leucine - an environmental modifier of insulin resistance acting on multiple levels of metabolism. PLoS One. 2011;6:e21187.

81. Hinault C, Mothe-Satney I, Gautier N, Lawrence JC Jr, Van Obberghen E. Amino acids and leucine allow insulin activation of the PKB/mTOR pathway in normal adipocytes treated with wortmannin and in adipocytes from $\mathrm{db}$ / db mice. FASEB J. 2004;18:1894-6.

82. Arakawa M, Masaki T, Nishimura J, Seike M, Yoshimatsu $H$. The effects of branched-chain amino acid granules on the accumulation of tissue triglycerides and uncoupling proteins in diet-induced obese mice. Endocr J. 2011;58:161-70.

83. Scaini $G$, Jeremias IC, Morais MO, Borges GD, Munhoz BP, Leffa DD, et al DNA damage in an animal model of maple syrup urine disease. Mol Genet Metab. 2012;106:169-74.

84. Kasperek GJ, Dohm GL, Snider RD. Activation of branched-chain keto acid dehydrogenase by exercise. Am J Phys. 1985;248:R166-71.

85. dos Santos RV, Caperuto EC, de Mello MT, Batista ML Jr, Rosa LF. Effect of exercise on glutamine synthesis and transport in skeletal muscle from rats. Clin Exp Pharmacol Physiol. 2009;36:770-5.

86. Shimomura Y, Fujii H, Suzuki M, Murakami T, Fujitsuka N, Nakai N. Branchedchain alpha-keto acid dehydrogenase complex in rat skeletal muscle: regulation of the activity and gene expression by nutrition and physical exercise. J Nutr. 1995;125:1762S-5S.

87. Poortmans JR, Siest G, Galteau MM, Houot O. Distribution of plasma amino acids in humans during submaximal prolonged exercise. Eur J Appl Physiol Occup Physiol. 1974;32:143-7.

88. Refsum HE, Gjessing LR, Strømme SB. Changes in plasma amino acid distribution and urine amino acids excretion during prolonged heavy exercise. Scand J Clin Lab Invest. 1979;39:407-13.
89. Ahlborg G, Felig P, Hagenfeldt L, Hendler R, Wahren J. Substrate turnover during prolonged exercise in man. Splanchnic and leg metabolism of glucose, free fatty acids, and amino acids. J Clin Invest. 1974;53:1080-90.

90. Shimomura Y, Murakami T, Nakai N, Nagasaki M, Harris RA. Exercise promotes BCAA catabolism: effects of BCAA supplementation on skeletal muscle during exercise. J Nutr. 2004;134:1583S-7S.

91. Spillane M, Emerson C, Willoughby DS. The effects of 8 weeks of heavy resistance training and branched-chain amino acid supplementation on body composition and muscle performance. Nutr Health. 2012;21:263-73.

92. Watson P, Shirreffs SM, Maughan RJ. The effect of acute branched-chain amino acid supplementation on prolonged exercise capacity in a warm environment. Eur J Appl Physiol. 2004;93:306-14.

93. Falavigna $\mathrm{G}$, de Araújo AJ, Rogero MM, Pires IS, Pedrosa RG, Martins $E$, et al. Effects of diets supplemented with branched-chain amino acids on the performance and fatigue mechanisms of rats submitted to prolonged physical exercise. Nutrients. 2012;4:1767-80.

94. Nawabi MD, Block KP, Chakrabarti MC, Buse MG. Administration of endotoxin, tumor necrosis factor, or interleukin 1 to rats activates skeletal muscle branched-chain a-keto acid dehydrogenase. J Clin Invest. 1990;85:256-63.

95. Fürst $P$, Albers $S$, Stehle P. Stress-induced intracellular glutamine depletion. The potential use of glutamine-containing peptides in parenteral nutrition. Beitr Infusionther Klin Ernahr. 1987:17:117-36.

96. Hardy G, Hardy IJ. Can glutamine enable the critically ill to cope better with infection? JPEN J Parenter Enteral Nutr. 2008;32:489-91.

97. Holecek M, Sispera L. Glutamine deficiency in extracellular fluid exerts adverse effects on protein and amino acid metabolism in skeletal muscle of healthy, laparotomized, and septic rats. Amino Acids. 2014;46:1377-84.

98. Hasselgren PO, Pedersen P, Sax HC, Warner BW, Fischer JE. Current concepts of protein turnover and amino acid transport in liver and skeletal muscle during sepsis. Arch Surg. 1988;123:992-9.

99. Gardiner K, Barbul A. Intestinal amino acid absorption during sepsis. JPEN J Parenter Enteral Nutr. 1993;17:277-83.

100. Bower RH, Kern KA, Fischer JE. Use of a branched chain amino acid enriched solution in patients under metabolic stress. Am J Surg. 1985;149:266-70.

101. Oki JC, Cuddy PG. Branched-chain amino acid support of stressed patients. DICP. 1989;23:399-410

102. Jiménez Jiménez FJ, Ortiz Leyba C, Morales Ménedez S, Barros Pérez M, Muñoz GJ. Prospective study on the efficacy of branched-chain amino acids in septic patients. J Parenter Enter Nutr. 1991;15:252-61.

103. De Bandt JP, Cynober L. Therapeutic use of branched-chain amino acids in burn, trauma, and sepsis. J Nutr. 2006;136:308S-13S.

104. Platell C, Kong SE, McCauley R, Hall JC. Branched-chain amino acids. J Gastroenterol Hepatol. 2000;15:706-17.

105. Mattick JSA, Kamisoglu K, lerapetritou MG, Androulakis IP, Berthiaume F. Branched-chain amino acid supplementation: impact on signaling and relevance to critical illness. Wiley Interdiscip Rev Syst Biol Med. 2013;5:449-60.

106. Lang $\mathrm{CH}$, Frost RA. Endotoxin disrupts the leucine-signaling pathway involving phosphorylation of mTOR, 4E-BP1, and S6K1 in skeletal muscle. J Cell Physiol. 2005;203:144-55.

107. Cole JT, Mitala CM, Kundu S, Verma A, Elkind JA, Nissim I, Cohen AS. Dietary branched chain amino acids ameliorate injury-induced cognitive impairment. Proc Natl Acad Sci U S A. 2010;107:366-71.

108. Jeter CB, Hergenroeder GW, Ward NH, Moore AN, Dash PK. Human mild traumatic brain injury decreases circulating branched-chain amino acids and their metabolite levels. J Neurotrauma. 2013;30:671-9.

109. Aquilani $R$, ladarola $P$, Contardi $A$, Boselli M, Verri M, Pastoris $O$, et al. Branched-chain amino acids enhance the cognitive recovery of patients with severe traumatic brain injury. Arch Phys Med Rehabil. 2005;86:1729-35.

110. Baracos VE, Mackenzie ML. Investigations of branched-chain amino acids and their metabolites in animal models of cancer. J Nutr. 2006;136:237S-42S.

111. Ananieva EA, Wilkinson AC. Branched-chain amino acid metabolism in cancer. Curr Opin Clin Nutr Metab Care. 2018;21:64-70.

112. Choudry HA, Pan M, Karinch AM, Souba WW. Branched-chain amino acid-enriched nutritional support in surgical and cancer patients. J Nutr. 2006:136:314S-8S 